\title{
BMJ Open Implementation of an evidence-based model of care for low back pain in emergency departments: protocol for the Sydney Health Partners Emergency Department (SHaPED) trial
}

To cite: Machado GC, Richards B, Needs C, et al. Implementation of an evidencebased model of care for low back pain in emergency departments: protocol for the Sydney Health Partners Emergency Department (SHaPED) trial. BMJ Open 2018;8:e019052. doi:10.1136/ bmjopen-2017-019052

- Prepublication history and additional material for this paper are available online. To view these files, please visit the journal online (http://dx.doi. org/10.1136/bmjopen-2017019052).

Received 8 August 2017 Revised 30 January 2018 Accepted 28 February 2018

Check for updates

For numbered affiliations see end of article.

Correspondence to Dr Gustavo C Machado; gustavo.machado@sydney. edu.au

\section{ABSTRACT}

Introduction Patients with low back pain often seek care in emergency departments, but the problem is that many patients receive unnecessary or ineffective interventions and at the same time miss out on the basics of care, such as advice on self-management. This pattern of care has important consequences for the healthcare system (expensive and inefficient) and for patients (poor health outcomes). We hypothesised that the implementation of an evidence-based model of care for low back pain will improve emergency care by reducing inappropriate overuse of tests and treatments and improving patient outcomes.

Methods and analysis A stepped-wedge cluster randomised controlled trial will be conducted to implement and evaluate the use of the Agency for Clinical Innovation (ACl) model of care for acute low back pain at four emergency departments in New South Wales, Australia. Clinician participants will be emergency physicians, nurses and physiotherapists. Codes from the Systematised Nomenclature of Medicine-Clinical Terms-Australian version will be used to identify low back pain presentations. The intervention, targeting emergency clinicians, will comprise educational materials and seminars and an audit and feedback approach. Health service delivery outcomes are routinely collected measures of imaging (primary outcome), opioid use and inpatient admission. A random subsample of 200 patient participants from each trial period will be included to measure patient outcomes (pain intensity, physical function, quality of life and experience with emergency service). The effectiveness of the intervention will be assessed by comparing the postintervention period with the retrospective baseline control period.

Ethics and dissemination The study received ethical approval from the Sydney Local Health District (Royal Prince Alfred Hospital zone) Ethics Committee (X17-0043). The results of this study will be published in peer-reviewed journals and presented at international conferences.

Trial registration number Australia New Zealand Clinical Trials Registry: ACTRN 12617001160325.

\section{Strengths and limitations of the study}

- This is a novel implementation trial looking at reduc ing inappropriate overuse of tests and treatments for low back pain in emergency departments.

- The stepped-wedge design is particularly suited to interventions aiming to improve healthcare systems as all sites receive the intervention.

- In this study design, intervention effects are estimated from within-emergency department differences while controlling for time trends.

- The use of routinely collected measures reduces the burden of data collection of health service delivery outcomes in the emergency departments.

- Incorporation of only four clusters (emergency departments) in the trial may limit the generalisability of results to other health districts.

\section{INTRODUCTION}

Background and rationale

Low back pain is a common presenting complaint in emergency settings. In 20152016 alone, there were 104072 low back pain presentations to emergency departments in Australia, placing this condition among the top 10 reasons for emergency visits. ${ }^{1}$ This condition is also a common reason for emergency department presentations across the globe, accounting for $4.4 \%$ of all presentations. ${ }^{2}$ Unfortunately, many patients receive low-value care for their low back pain in the emergency department. Low-value care is broadly defined as the use of an intervention that provides patients with little to no benefits or causes harm. ${ }^{3}$ Examples of low-value care of low back pain in emergency departments include inappropriate overuse of imaging, liberal 
use of opioid analgesics and unnecessary admission to hospital.

Multiple clinical guidelines exist for the management of low back pain in primary care. ${ }^{45}$ Although it is unclear whether these guidelines should be applied in the emergency department, much of their recommendations may be relevant to emergency physicians and are often used to guide their practice. ${ }^{6}$ However, the mixture of providing inappropriate care and failing to provide appropriate care in the emergency department is a clear indication that healthcare is not following clinical guidelines. For instance, about $30 \%$ of patients with non-specific low back pain receive imaging in the emergency department ${ }^{7}$ when guidelines explicitly recommend no imaging for these cases. Imaging in the absence of suspected serious pathology does not improve patient outcomes ${ }^{8}$ and can potentially cause harms. ${ }^{9-11}$ Against guideline advice, around $62 \%$ of low back pain patients receive opioids in the emergency department, ${ }^{12}$ although efficacy in pain relief has not been established for acute low back pain ${ }^{13}$ and side effects are often serious, ${ }^{14}$ including dependence, overdose and death. Another issue is the increasing rate of hospital admissions. More than one-third of low back pain presentations to the emergency department lead to the patient being admitted to hospital, ${ }^{7}$ where care is likely to be similar to what could be provided in primary care.

The significant deviations from evidence-based recommendations occurring in Australian emergency departments ${ }^{15}$ make them an appropriate setting to trial an intervention based on improving care for low back pain. The Agency for Clinical Innovation (ACI) has recently launched a model of care for acute low back pain that could be applied in both primary care and emergency department settings. ${ }^{16}$ The ACI model of care was developed in collaboration with policy-makers, clinicians, consumers and researchers and distils the high-quality evidence in this area to formulate key messages for practice (table 1). Briefly, the model provides different care pathways according to a classification based on a diagnostic triage ${ }^{17}$ (acute or chronic non-specific low back pain, low back pain with leg pain and suspected serious spinal conditions). Risk stratification ${ }^{18}$ is recommended to guide the amount and type of treatment provided; including personalised evidence-based health education and treatment. Lastly, follow-up reviews are scheduled to monitor individuals' progress. Passive dissemination of guidelines, such as the ACI model of care, is unlikely to change practice. We are proposing a multifaceted strategy to implement and evaluate the ACI model of care to see if this improves health service delivery and patient outcomes for low back pain at the emergency department.

\begin{tabular}{|c|c|}
\hline Principle 1 & Assessment: history and examination \\
\hline Principle 2 & Risk stratification \\
\hline Principle 3 & Patient education \\
\hline Principle 4 & Active physical therapy encouraged \\
\hline Principle 5 & Begin with simple analgesic medicines \\
\hline Principle 6 & Judicious use of complex medicines \\
\hline Principle 7 & Cognitive behavioural approach \\
\hline Principle 8 & $\begin{array}{l}\text { Only image those with suspected serious } \\
\text { spinal pathology }\end{array}$ \\
\hline Principle 9 & Predetermined times for review \\
\hline Principle 10 & $\begin{array}{l}\text { Timely referral and access to specialist } \\
\text { services }\end{array}$ \\
\hline
\end{tabular}

Source: NSW Agency for Clinical Innovation. Management of people with acute low back pain: model of care. Chatswood: NSW Health, 2016. Available at: https://www.aci.health.nsw.gov.au/ resources/musculoskeletal/management-of-people-with-acutelow-back-pain/albp-model.

\section{OBJECTIVES}

The overall aim of the Sydney Health Partners Emergency Department (SHaPED) trial is to implement and evaluate the ACI model of care for acute low back pain. The outcomes of the trial reflect the key messages in the model: (1) patients with non-specific low back pain do not require imaging; (2) where medicines are used, simple analgesics should be the first option; (3) patients with non-specific low back pain should be managed as outpatients.

\section{Primary objective}

The primary objective of this study is to evaluate if implementation of the ACI model of care significantly reduces the proportion of patients presenting with low back pain who receive imaging in the emergency department.

\section{Secondary objectives}

The secondary aims of the study are:

- To determine if implementation of the ACI model of care significantly reduces the proportion of patients presenting with low back pain who receive opioids in the emergency department and the proportion of patients subsequently admitted to hospital.

- To determine if implementation of the ACI model of care significantly improves patient outcomes in people who present with low back pain in the emergency department.

- To determine the cost-effectiveness of the ACI model of care compared with current emergency department practice for people who present with low back pain.

- To determine the barriers and facilitators to the implementation intervention of the ACI model of care for people who present with low back pain in the emergency department. 
Table 2 SHaPED trial design

\begin{tabular}{|c|c|c|c|c|c|c|c|c|c|c|c|c|c|c|c|c|c|c|c|}
\hline \multirow{2}{*}{$\begin{array}{l}\text { Steps } \\
\text { (clusters) }\end{array}$} & \multicolumn{12}{|c|}{ Year 1} & \multicolumn{7}{|c|}{ Year 2} \\
\hline & 1 & 2 & 3 & 4 & 5 & 6 & 7 & 8 & 9 & 10 & 11 & 12 & 1 & 2 & 3 & 4 & 5 & 6 & 7 \\
\hline \multicolumn{20}{|l|}{ ED 1} \\
\hline \multicolumn{20}{|l|}{ ED 2} \\
\hline \multicolumn{20}{|l|}{ ED 3} \\
\hline ED 4 & & & & & & & & & & & & & & & & & & & \\
\hline
\end{tabular}

Light grey: 12-month retrospective baseline control period.

Middle grey: 4-week initial intervention period.

Dark grey: sites continue with intervention plus follow-up period.

ED, emergency department; SHaPED, Sydney Health Partners Emergency Department.

\section{METHODS AND ANALYSIS \\ Study design}

The Standard Protocol Items: Recommendations for Interventional Trials guidelines were followed in this report of the protocol. ${ }^{19}$ SHaPED will use a steppedwedge cluster randomised controlled trial design. ${ }^{20}$ In this study design, clusters are randomised to cross from the control period (ie, unexposed to intervention) to the intervention period at regular intervals ('steps') until all clusters have crossed to the intervention under evaluation. This design is particularly suited to interventions aiming to improve healthcare systems as all groups eventually receive the intervention. Moreover, the process allows for comparison with control sites that have not yet implemented the intervention.

In the SHaPED trial, after a retrospective baseline observation control period of 12 months prior to randomisation, the intervention will be sequentially rolled out, with a new emergency department receiving the intervention every 4 weeks, until all participating emergency departments have received the intervention. After the implementation of the ACI model of care, the emergency departments will continue using the pathways of care outlined in the model until the end of the trial (table 2).

\section{Study setting}

The emergency departments of one rural and three urban hospitals in New South Wales, Australia will participate in the study: Royal Prince Alfred Hospital, Concord Repatriation General Hospital, Canterbury Hospital and Dubbo Base Hospital. Investigators in the SHaPED trial are listed in online supplementary appendix 1 .

\section{Clinician participants}

Clinician participants included in the SHaPED trial will be emergency clinical staff, such as physicians, nurses and physiotherapists, who routinely manage patients presenting to emergency departments with a primary complaint of low back pain. Potential clinician participants will be invited by the principal investigator of each emergency department and will receive a participant information statement. Research staff will verbally explain the information provided in this document to fully inform potential clinician participants of the risks and benefits of their participation. In addition, the research staff will be available to answer any questions to ensure that potential clinician participants fully understand the implications of their decision. A written participant consent form will be obtained from all participating clinicians prior to randomisation.

\section{Patient participants}

We will use codes from the Systematised Nomenclature of Medicine-Clinical Terms-Australian version, Emergency Department Reference Set ${ }^{21}$ to identify low back pain presentations (online supplementary appendix 2) to the emergency departments. Presentations with codes related to low back pain with non-specific cause or those associated with neurological signs and symptoms (such as sciatica and lumbar spinal stenosis) will be included. Representations to the emergency department within 48 hours or low back pain presentations related to serious spinal pathologies (such as lumbar fracture or cauda equina syndrome) will be excluded. A random subsample of 200 patient participants from each trial period will be referred to a brief self-reported online questionnaire to evaluate the effectiveness of the implementation of the ACI model of care on patient outcomes.

\section{Randomisation}

Before the beginning of the intervention, the four hospitals will be randomly allocated the 'step' when the intervention will commence at their emergency department. Randomisation will be conducted using computer-generated random numbers by research staff. Only the research team will be aware of cluster allocation.

\section{Intervention}

A framework has been proposed to facilitate the implementation of research evidence into clinical practice, known as the knowledge-to-action process. ${ }^{22}$ This framework links the various types of research enquiry with the key steps in the research translation cycle. The process consists of the knowledge creation cycle and the action cycle and involves end users of research (eg, policy-makers, clinicians and patients) to facilitate engagement with the implementation strategy. We will use this framework to develop a tailored intervention strategy to 
implement the ACI model of care at the participating emergency departments.

Implementation will begin with visits to each participating emergency department to establish collaborations and approvals. We will also assess organisational issues and potential barriers to the implementation intervention, such as intake and flow of patients with low back pain, assessment of current practices, acceptability of new model and specific roles of emergency clinicians in managing these patients. We will identify existing models of care that are used to guide management of patients presenting with low back pain at each emergency department. Then, we will work with local clinical staff to ensure that each site practices according to the full ACI model of care.

A multifaceted intervention package will be used to implement the ACI model of care at the emergency departments. Briefly, the initial 4-week intervention will consist of printed and electronic educational materials, educational seminars and educational outreach, website support, posters and an audit and feedback approach. Clinician participants will receive a copy of the model and other printed materials, including the ACI consumer information booklet, as well as access to additional online support tools outlined in the ACI model of care, such as web pages and videos, to help them educate their patients. Experienced clinicians, research staff and local opinion leaders (ie, directors of emergency medicine) will deliver the interactive educational seminars and educational outreach. An audit and feedback approach focused on the outcomes of the study will also be used to enhance our implementation programme. A detailed description of the implementation plan for the SHaPED trial can be found in online supplementary appendix 3 .

The intervention will be tailored for each site by adapting knowledge resources (such as printed decision aids and patient resources) to the local context and by working with local opinion leaders to address potential barriers to implementing the ACI model of care. These instructions, measures and training materials will be hosted online during the implementation phase on The University of Sydney's website. Due to the nature of the intervention, it will not be possible to blind clinician participants to the intervention.

\section{Sample size}

Based on the effect size of $10 \%$ absolute reduction (from $30 \%^{7}$ to $20 \%$ ) in imaging referrals, combined with an alpha of 0.05 and assuming an intraclass correlation coefficient of 0.1, a total number of 1920 low back pain presentations (on average 480 per cluster) to emergency departments is needed for this stepped-wedge cluster trial with $80 \%$ power. A preliminary analysis revealed that there were over 2650 low back pain presentations to the participating emergency departments in 2016, showing feasibility of this trial.

\begin{tabular}{ll}
\hline Table 3 & Medications per ATC classification \\
\hline Group & ATC code \\
\hline Analgesics & N02B \\
NSAIDs & M01A \\
& M02AA \\
Muscle relaxants & M03 \\
Opioids & N02A \\
& N01AH \\
Neuropathic pain medicines & N03 \\
& N06A
\end{tabular}

ATC, Anatomical Therapeutic Chemical; NSAIDs, non-steroidal anti-inflammatory drugs.

\section{Outcome measures}

Clinician participants will complete a baseline questionnaire, including demographic questions. They will also be asked to indicate whether they have special interests in low back pain or musculoskeletal medicine, and if they had attended previous continuing medical education or postgraduate training on low back pain management. The outcomes to evaluate the effectiveness of the ACI model of care on health service delivery are routinely collected emergency department measures.

\section{Primary outcome}

- Proportion of patients receiving any imaging (yes/ no).

\section{Secondary outcomes}

- Proportion of patients receiving advanced imaging (CT $/ \mathrm{MRI}=y e s, \mathrm{X}$-ray/no imaging=no).

- Proportion of patients receiving analgesic medications (topical, oral, injection). Medications will be classified according to the Anatomical Therapeutic Chemical (ATC) classification system (table 3). The ATC classification is recommended by the WHO and is widely used internationally in medication use studies:

- Paracetamol.

- Non-steroidal anti-inflammatory drugs.

- Muscle relaxants.

- Opioids.

- Neuropathic pain medications.

- Other.

- Proportion of patients admitted to:

- Hospital.

- Emergency Medical Unit.

- Short Stay Unit.

- Time in emergency department (triage time to discharge or admission time).

- Proportion of patients referred to specialists (referral for a consultation by the emergency department):

- Pain management.

- Rheumatology.

- Surgery.

- Proportion of patients representing to the emergency department within 48 hours. 
- Proportion of patients readmitted to the hospital within 28 days.

- Total health system costs (including intervention costs and health service delivery costs).

Patient outcomes will be collected using a brief online questionnaire that will measure pain intensity (Numeric Rating Scale, range 0-10). We will also use the Patient-Reported Outcomes Measurement Information System (PROMIS) to measure physical function (PROMIS Short Form-Physical Function 4a) and quality of life (PROMIS Scale-Global Health item 1) as advocated by the National Institutes of Health. We have chosen these outcomes as they are considered the three core outcome domains for clinical trials in low back pain identified in a recent Delphi study ${ }^{23}$ and by the International Consortium for Health Outcomes Measurement. ${ }^{24}$ Patient experience with emergency service will be assessed using item 31 of the Emergency Department Patient Experience of Care survey advocated by the American College of Emergency Medicine. ${ }^{25}$

\section{Data collection methods}

In the week prior to the implementation intervention, the 12-month retrospective baseline health service delivery data will be extracted directly from participating hospitals' electronic record systems. The Sydney Local Health District (SLHD) Targeted Activity and Reporting System (STARS) will be used to access and extract data from SLHD emergency departments. STARS is data analytics programme which monitors clinician performance and service use. At Dubbo Base Hospital, health service delivery data will be extracted from its electronic record system. During the intervention, health service delivery measures will be extracted from all participating emergency departments every week until the end of the 3-month follow-up period. Data extraction will be conducted remotely for all participating emergency departments by research staff blinded to intervention allocation. Data collection through hospitals' electronic systems will also avoid additional workloads within the emergency departments.

Patient outcome measures will be collected using automated text messaging at 1 week (primary time point) and again at 2 and 4 weeks after index emergency department presentation. A random subsample of patient participants will be referred to a brief self-reported online questionnaire containing the Patient Information Statement. Completion of the online questionnaire indicates patient consent to participate in the study. Three reminder messages will be sent to non-responders, and those who do not respond to the third message will be contacted verbally via telephone.

Data will be securely stored in password-protected spreadsheets and transferred to appropriate statistical software for analysis. Spreadsheets will be regularly scrutinised for omissions and errors. Data will be archived at the Sydney School of Public Health, The University of Sydney for 15 years, after which data will be destroyed.

\section{Statistical methods}

Data analysis will be performed according to an intention-to-treat analysis, that is, clusters will be analysed according to their randomised cross-over time irrespective of whether cross-over was achieved at the desired time. First, we will investigate temporal trends in healthcare outcomes across the 12-month baseline observation period. In the situation of an underlying temporal trend, we will only include data for the previous 3 months as the baseline observation period. In our primary analysis, the 4-week implementation intervention period will be excluded, but a secondary exploratory analysis will be performed including the implementation period into the intervention group. For the primary outcome analysis, logistic regression models with a random effect for cluster, a fixed effect indicating the group assignment of each cluster at each step and a fixed effect of time (each step) will be used. Data will be analysed using SAS V.9.1.3 (SAS Institute).

\section{Economic evaluation}

An economic evaluation of the ACI model of care compared with current emergency practice will be undertaken from the health system perspective. First, we will measure the costs related to the delivery of the intervention (that is, training component, staff time and printed resources). Then, the costs related to health service delivery will be measured via data captured by the hospitals' electronic record systems. Costs will be valued based on government charges, using publicly available data. All costs will be reported in Australian dollars. Where necessary, costs will be converted to 2018 prices using the Australian Institute of Health and Welfare (AIHW) health price index. The incremental cost-effectiveness ratio (ICER) will be presented as the incremental cost per patient avoiding any imaging, opioid prescription and hospital admission.

Univariate sensitivity analyses will be conducted around key parameters likely to influence cost-effectiveness, including cost and efficacy estimates. For example, effectiveness parameters used in the economic evaluation will be varied over the 95\% CIs to assess impact on the ICER. Intervention costs, including training costs, staff time and resource costs will be collected from individual emergency departments and similarly, analysis will examine the effect on the ICER of varying these values over the range reported by participating sites. Bootstrapping will be used to estimate a distribution around costs and health outcomes and to estimate the CIs around the ICER. Results will be plotted on the cost-effectiveness plane.

\section{Process evaluation}

A process evaluation will be conducted to provide an indication of which elements of the intervention are effective and worthwhile. In the week before the implementation period and in the week after it, clinician participants will be asked to answer a questionnaire containing the Back Beliefs Questionnaire. ${ }^{26}$ The Back Beliefs Questionnaire 
is a widely validated questionnaire ${ }^{27}$ designed to measure beliefs about low back pain and will be used in our trial to assess whether the use of the ACI model of care improves beliefs about low back pain among emergency clinicians. This instrument was found to be reliable and responsive to change in a wide range of contexts, including in Australia. ${ }^{28}$ We will also use a set of questions aimed at eliciting knowledge about the management of low back pain and attitudes of emergency clinicians towards these patients. ${ }^{29}$ At the end of the implementation period, clinician participants will also be asked to review the content of educational materials. Potential barriers and facilitators will be investigated using qualitative interviews with clinician participants.

\section{ETHICS AND DISSEMINATION}

Our hypothesis is that implementation of the ACI model of care will improve health service delivery in participating emergency departments for patients presenting with low back pain: specifically decreasing the proportion of patients receiving imaging, opioids and hospital admission. If the trial results are positive, we will build on our existing strong relationships with the ACI, Sydney Health Partners and the Local Health Districts to support implementation of the ACI model of care in other emergency departments across New South Wales. As a branch of the New South Wales Ministry of Health, the ACI will be well positioned to facilitate transferability of findings. We will also disseminate the results of the trial at conferences and in scientific journals, and we will continue our successful approach of using the media to reach a lay audience and health consumers. The study resources will be made freely available on relevant websites so that jurisdictions beyond New South Wales can adopt the implementation strategy outlined in this study.

\section{Author affiliations}

${ }^{1}$ Sydney School of Public Health, The University of Sydney, Sydney, New South Wales, Australia

${ }^{2}$ Institute for Musculoskeletal Health, Sydney Local Health District, Sydney, New South Wales, Australia

${ }^{3}$ Rheumatology Department, Royal Prince Alfred Hospital, Camperdown, New South Wales, Australia

${ }^{4}$ Monash Department of Clinical Epidemiology, Cabrini Institute, Melbourne, Victoria, Australia

${ }^{5}$ Department of Epidemiology and Preventive Medicine, School of Public Health and Preventive Medicine, Monash University, Melbourne, Victoria, Australia

${ }^{6}$ South Western Sydney Clinical School, University of New South Wales, Sydney, New South Wales, Australia

${ }^{7}$ The George Institute for Global Health, University of New South Wales, Sydney, New South Wales, Australia

${ }^{8}$ Emergency Department, Royal Prince Alfred Hospital, Sydney, New South Wales, Australia

${ }^{9}$ Emergency Department, Canterbury Hospital, Sydney, New South Wales, Australia

${ }^{10}$ Emergency Department, Concord Repatriation General Hospital, Sydney, New

South Wales, Australia

${ }^{11}$ Emergency Department, Dubbo Base Hospital, Dubbo, New South Wales, Australia

Collaborators Matthew Oliver; Danielle Coombs; Matthew Chu; Daniel Harrison; Mauricio Oliveira; Noel Baidya; Hannah Storey; Rachael Knoblanche; Matthew
Jennings; Robyn Speerin; Nobby Alcala; Niamh Moloney; Manuela Ferreira; Paulo Ferreira; Chris Lin.

Contributors GCM, BR, CN, RB, IH, KH, KM, LB and CGM conceptualised the research design, drafted the research protocol and are coordinating with the project team. JE, ER, RF, DLC provided expert advice and are lead site investigators. M0, $\mathrm{NB}, \mathrm{HS}$ and RK are responsible for the acquisition of data and data monitoring. MJ, $\mathrm{RS}, \mathrm{NA}, \mathrm{NM}, \mathrm{MF}, \mathrm{PF}$ and $\mathrm{CL}$ are collaborators and contributed with expert advice and funding applications. LB advised on the trial design and was responsible for the sample size calculation and statistical analysis methods. KH was responsible for the design of the economic evaluation. KM was responsible for the design of the process evaluation. $\mathrm{MO}, \mathrm{DC}, \mathrm{MC}$, and $\mathrm{DH}$ are site investigators contributing to the implementation of the model of care. All authors contributed to refinement of the study protocol and approved the final manuscript.

Funding The SHaPED trial received seed funding (\$A90 000) from Sydney Health Partners. GCM is funded by a National Health and Medical Research Council (NHMRC) Early Career Fellowship. RB is funded by an NHMRC Senior Principal Research Fellowship. CGM is funded by an NHMRC Principal Research Fellowship. Study sponsor: The University of Sydney, NSW 2006 Australia.

Competing interests None declared.

Patient consent Not required.

Ethics approval The study received ethical approval from the Sydney Local Health District (RPAH zone) Ethics Committee, Sydney, Australia (X17-0043).

Provenance and peer review Not commissioned; externally peer reviewed.

Open Access This is an Open Access article distributed in accordance with the Creative Commons Attribution Non Commercial (CC BY-NC 4.0) license, which permits others to distribute, remix, adapt, build upon this work non-commercially, and license their derivative works on different terms, provided the original work is properly cited and the use is non-commercial. See: http://creativecommons.org/ licenses/by-nc/4.0/

(C) Article author(s) (or their employer(s) unless otherwise stated in the text of the article) 2018. All rights reserved. No commercial use is permitted unless otherwise expressly granted.

\section{REFERENCES}

1. Australian Institute of Health and Welfare. Emergency department care 2015-16: Australian hospital statistics. Canberra: Australian Institute of Health and Welfare, 2016.

2. Edwards J, Hayden J, Asbridge M, et al. Prevalence of low back pain in emergency settings: a systematic review and meta-analysis. BMC Musculoskelet Disord 2017;18:143.

3. Scott IA, Duckett SJ. In search of professional consensus in defining and reducing low-value care. Med J Aust 2015;203:179-81.

4. Qaseem A, Wilt TJ, McLean RM, et al. Noninvasive treatments for acute, subacute, and chronic low back pain: a clinical practice guideline from the american college of physicians. Ann Intern Med 2017;166:514.

5. National Institute for Health and Care Excellence. Low back pain and sciatica in over 16s: assessment and management: NICE Guideline (NG59). 2016.

6. Edlow JA. Managing nontraumatic acute back pain. Ann Emerg Med 2015;66:148-53.

7. McCaughey EJ, Li L, Georgiou A, et al. Imaging for patients presenting to an emergency department with back pain: impact on patient pathway. Emerg Med Australas 2016;28:412-8.

8. Jarvik JG, Gold LS, Comstock BA, et al. Association of early imaging for back pain with clinical outcomes in older adults. JAMA 2015;313:1143-53.

9. Webster BS, Cifuentes M. Relationship of early magnetic resonance imaging for work-related acute low back pain with disability and medical utilization outcomes. J Occup Environ Med 2010;52:900-7.

10. Sloan TJ, Walsh DA. Explanatory and diagnostic labels and perceived prognosis in chronic low back pain. Spine 2010;35:E112 0-E1125.

11. Chou R, Qaseem A, Owens DK, et al. Diagnostic imaging for low back pain: advice for high-value health care from the American College of Physicians. Ann Intern Med 2011;154:181-9.

12. Friedman BW, Chilstrom M, Bijur PE, et al. Diagnostic testing and treatment of low back pain in United States emergency departments: a national perspective. Spine 2010;35:E1406-E1411.

13. Abdel Shaheed C, Maher CG, Williams KA, et al. Efficacy, tolerability, and dose-dependent effects of opioid analgesics for low back 
pain: a systematic review and meta-analysis. JAMA Intern Med 2016;176:958-68.

14. Dowell D, Haegerich TM, Chou R. CDC guideline for prescribing opioids for chronic pain-United States, 2016. JAMA 2016;315:1624-45.

15. Machado GC, Rogan E, Maher CG. Managing non-serious low back pain in the emergency department: Time for a change? Emerg Med Australas 2017.

16. NSW Agency for Clinical Innovation. Management of people with acute low back pain: model of care. Chatswood 2016.

17. Maher C, Underwood M, Buchbinder R. Non-specific low back pain Lancet 2017;389:736-47.

18. Hill JC, Whitehurst DG, Lewis M, et al. Comparison of stratified primary care management for low back pain with current best practice (STarT Back): a randomised controlled trial. Lancet 2011;378:1560-71.

19. Chan AW, Tetzlaff JM, Altman DG, et al. SPIRIT 2013 statement: defining standard protocol items for clinical trials. Ann Intern Med 2013;158:200-7.

20. Hemming K, Haines TP, Chilton PJ, et al. The stepped wedge cluster randomised trial: rationale, design, analysis, and reporting. BMJ 2015;350:h391.

21. Hansen DP, Kemp ML, Mills SR, et al. Developing a national emergency department data reference set based on SNOMED CT. Med J Aust 2011;194:S8-10.
22. Graham ID, Logan J, Harrison MB, et al. Lost in knowledge translation: time for a map? J Contin Educ Health Prof 2006;26:13-24.

23. Chiarotto A, Deyo RA, Terwee CB, et al. Core outcome domains for clinical trials in non-specific low back pain. Eur Spine $J$ 2015;24:1127-42.

24. Clement RC, Welander A, Stowell C, et al. A proposed set of metrics for standardized outcome reporting in the management of low back pain. Acta Orthop 2015;86:523-33.

25. Weinick RM, Becker K, Parast L, et al. Emergency department patient experience of care survey: development and field test. Rand Health Q 2014;4:5.

26. Symonds TL, Burton AK, Tillotson KM, et al. Do attitudes and beliefs influence work loss due to low back trouble? Occup Med 1996;46:25-32

27. Bostick GP, Schopflocher D, Gross DP. Validity evidence for the back beliefs questionnaire in the general population. Eur J Pain 2013;17:1074-81.

28. Buchbinder R, Jolley D, Wyatt M. Population based intervention to change back pain beliefs and disability: three part evaluation. BM 2001;322:1516-20.

29. Buchbinder R, Staples M, Jolley D. Doctors with a special interest in back pain have poorer knowledge about how to treat back pain Spine 2009;34:1218-26. 